\title{
Comparison between the in-vitro cytotoxicity of three different multilayer thermoplastic clear aligner materials
}

\author{
Spencer Marsh ${ }^{1,}{ }^{*}$, Ravikumar Anthony ${ }^{2}$, Blair Barnett ${ }^{3}$, Chen Shou ${ }^{4}$, Kristin Saunders ${ }^{3}$ \\ ${ }^{1}$ Resident, ${ }^{2}$ Orthodontic Program Director, ${ }^{3}$ Orthodontic Part-time Faculty, Department of Developmental Dentistry, Division of Orthodontics, \\ University of Texas Health Science Center at San Antonio, United States. \\ ${ }^{4}$ Pediatric Full-time Faculty, Department of Developmental Dentistry, Division of Orthodontics, University of Texas Health Science Center at San \\ Antonio, United States.
}

\begin{tabular}{l} 
Article History \\
Received $20^{\text {th }}$ April 2021 \\
Accepted $3^{\text {rd }}$ September2021 \\
Available online \\
$6^{\text {th }}$ February 2022 \\
*Correspondence \\
\hline Spencer Marsh DDS, MS \\
Postdoctoral Program in Orthodontics \\
UT Health San Antonio \\
8210 Floyd Curl Drive, MC 8115, San Antonio, \\
TX 78229-3923. \\
E-mail: spencermarshdds@gmail.com \\
Dol: $\underline{\text { http://dx.doi.org/10.37983/IIDM.2022.4101 }}$
\end{tabular}

\begin{abstract}
Background: Clear aligner therapy (CAT) is a prominent orthodontic treatment option. CAT was formerly only used to treat mild malocclusions, but with developments in technology, it can now treat much more complex malocclusions. With the increasing popularity of CAT and technological improvements, led to the development of Invisalign's SmartTrack technology, the first commercially available aligner material that used multi-layer plastic to facilitate tooth movement. Multiple layers provide superior mechanical properties that eluded previous single layer plastics.

Aim: To study the cytotoxicity properties of different thermoplastic multilayer clear aligner materials on human primary gingival fibroblasts (HGFs).

Materials and methods: Three multilayered clear aligner materials were considered in this study: SmartTrack (Align Technology, San Jose, CA, USA), Zendura FLX (Bay Materials, Fremont, CA, USA), and ComfortTrack (Great Lakes Dental Technologies, Tonawanda, NY, USA). The samples were incubated at $37^{\circ} \mathrm{C}$ in DMEM $(0.1 \mathrm{mg} / \mathrm{mL})$ for 21 days. The cell viability of HGFs cultured with each sample medium was then compared to a negative control assessed by MTT assay.

Results: The results showed slight toxicity for each one of the samples tested. The highest cytotoxicity level seen in the HGFs was SmartTrack $(65.5 \% \pm 2.5$ of cell viability), followed by Zendura FLX $(72.3 \% \pm 8.6)$, and the least was observed by ComfortTrack $(80.8 \% \pm 2.1)$.

Conclusion: The Under the experimental conditions of the study, all of the materials tested displayed slight levels of cytotoxicity. SmartTrack was measured as the most cytotoxic. There were no statistical differences found between the three aligner materials $(P<0.05)$.

Keywords: Clear Aligners, Cytotoxicity, Invisalign, Fibroblasts.
\end{abstract}

\section{Introduction}

Clear aligner therapy (CAT) has become a popular choice for orthodontic treatment. As more adults are seeking orthodontic treatment, there is a corresponding demand for esthetic treatment options [1-3]. Even children and adolescents show preference to orthodontic appliances that are less visible than traditional metal brackets and wires [4]. Other advantages of CAT over fixed appliances are fewer emergencies, shorter treatment visits, [5] and greater patient comfort [6].

As CAT's popularity has increased, so too has the technology improved. Most notable over the last decade is Invisalign's SmartTrack technology (Align Technology, San Jose, CA, USA). Released in 2013, it was the first commercially available aligner material that used multilayer plastic to support tooth movement and has become preferred over previous materials by both clinicians and patients [7]. Multiple layers offer superior mechanical properties that eluded previous single layer plastics [8]. CAT was originally only used for the correction of minor malocclusions, but with technological advances, they now can treat much more complex malocclusions $[9,10]$. As a result of this, treatment times are increasing, and patients are required to wear their clear aligners for longer durations. Each stage of aligners is meant to be worn for about 22 hours a day for up to 21 days each. It has been shown that the thickness of the aligners can be diminished after exposure to the oral cavity over this time; [11] it may be possible that harmful molecules are being released into the oral cavity. Align Technologies shipped over 1.2 million cases in 2018, [12] thus the importance of investigating this issue further as millions of people are treated with CAT worldwide each year. There has been extensive research on the biocompatibility of other orthodontic materials such as brackets, [13] wires, [14] and adhesives, [15] but only three previous papers have focused on the potential toxicity of clear aligner plastics [1618]. Furthermore, there have been several new multi-layer 
plastics introduced to the market that have not yet been publicly tested.

The purpose of this study, therefore, was to test the cytotoxicity of 3 different multilayer thermoplastic materials - SmartTrack (Align Technology, San Jose, CA, USA), Zendura FLX (Bay Materials, Fremont, CA, USA), and ComfortTrack (Great Lakes Dental Technologies, Tonawanda, NY, USA).

\section{Materials and Methods}

The three materials that were evaluated in this study were SmartTrack (Align Technology, San Jose, CA, USA), Zendura FLX (Bay Materials, Fremont, CA, USA), and ComfortTrack (Great Lakes Dental Technologies, Tonawanda, NY, USA). The cytotoxicity of each material was tested after they had been thermoformed.

Each sample material was sterilized following the protocol defined by the International Standards (ISO) 10993-5 norm. The samples were immersed in Dulbecco's Modified Eagles Medium (DMEM) and stored under stationary conditions at $37^{\circ} \mathrm{C}$ in airtight test tubes for 21 days. The ratio between the weight of the samples and the volume of the dilutions was $0.1 \mathrm{~g} / \mathrm{mL}$ as recommended by ISO parameters. After the release interval, the extracts were sterile-filtered to eliminate solid particles and stored at $20^{\circ} \mathrm{C}$ until further use.

MTT assay [3-(4,5-dimethylthiazol-2-yl)-2,5-diphenyl tetrazolium bromide] was used to evaluate cell viability. Human gingival fibroblasts (HGFs) were plated into 48well flat-bottomed, tissue culture plates, with a density of 208 cells/well. After 24 hours of incubation, the culture medium was replaced with $400 \mu \mathrm{L} /$ well of clear aligner extract. After an additional 24 hours, the medium was replaced with $200 \mu \mathrm{L} /$ well of the MTT solution ( $1 \mathrm{mg} / \mathrm{mL}$ ), mixed gently for 10 minutes, and the cells were incubated for an additional four hours at $37^{\circ} \mathrm{C}$. $200 \mu \mathrm{L}$ of isopropanol with $0.04 \mathrm{~N} \mathrm{HCL}$ was then added to each well and mixed thoroughly by repeated pipetting. The optical density of each sample was measured in a spectrophotometer at $600 \mathrm{~nm}$. The optical density (OD) of the cells cultured in the DMEM medium without any clear aligner material sample extracts was used as a negative control for $100 \%$ cell viability and as a reference for the determination of the level of cytotoxicity in the assay. Cells treated with a $1 \%$ concentration of anionic detergent were used as a positive control [21].

\subsection{Analysis}

The optical density of each sample was used to calculate the cell viability using the following criteria:

- $\quad$ Cell viability $=$ (optical density of test group $/$ optical density of cellular control group) X 100 as calculated by Vande Vannet et al. (2006) [20].

- Cell viability was scored using the classification of Sjögren et al. (2000) [19]:

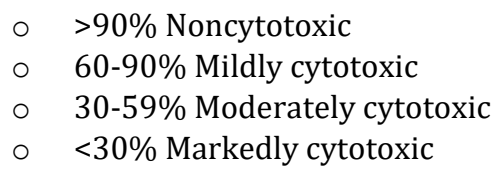

Descriptive statistics and statistical analysis were performed using the Statistical Package for Social Sciences (SPSS 27.0, SPSS IBM, Armonk, NY, USA). The normal distribution of the data was confirmed by the Shapiro-Wilk test at $\mathrm{p}>0.05$. Differences between mean values were determined by a oneway Welch analysis of variance (ANOVA) with the GamesHowell post hoc test at $\mathrm{p}<0.05$.

\section{Results}

The MTT assay is a colorimetric assay used to measure cellular metabolic activity as an indicator of cell viability. The yellow tetrazolium salt is converted to purple formazan crystals by mitochondrial reductase enzymes in metabolically active cells. The resulting-coloured solution is quantified by measuring the absorbance at 600 nanometers using a multi-well spectrometer. The greater number of viable cells, the darker the solution will be $[29,30]$.

The raw OD readings from each of the materials are listed in Figure 1. All three samples showed significant differences when compared to both the negative and positive controls, but not when compared to one another. The cytotoxicity levels of each sample after the viability formula were applied (Cell viability $=$ (optical density of test group/ optical density of cellular control group) X 100) is shown in table 1 . All the tested plastics showed a mildly cytotoxic influence on the HGFs after 21 days of incubation.

The material that expressed the highest cytotoxicity was SmartTrack $(65.5 \% \pm 2.5$ of cell viability), followed by Zendura FLX $(72.3 \% \pm 8.6)$, and the least was observed by ComfortTrack $(80.8 \% \pm 2.1)$. The positive control resulted in the viability of only $12.4 \% \pm 0.8$.

Table 1. Mean viability and standard deviation, cytotoxicity levels of materials.

\begin{tabular}{cccc}
\hline Materials & $\begin{array}{c}\text { Mean } \\
\text { Viability } \pm \\
\text { SD }^{\ddagger}\end{array}$ & Cytotoxicity & Significance \\
\hline Zendura & $72.3 \pm 8.6$ & Mild & NS* \\
\hline Great Lakes & $80.8 \pm 2.1$ & Mild & NS* $^{*}$ \\
\hline SmartTrack & $65.5 \pm 2.5$ & Mild & NS* $^{*}$ \\
\hline
\end{tabular}

${ }^{\text {}}$ Standard deviation, ${ }^{*}$ No significant difference

\section{Discussion}

With the increasing popularity of CAT and the expiration of patents previously held exclusively by Align Technology, the orthodontic industry is currently seeing a flood of new aligner materials being introduced into the market. In addition, the accessibility of digital scanning and 3D printing have made it easier than ever for dental professionals to 
provide effective in-office treatments without relying on large external labs. It is imperative that these treatments be administered in a manner that is efficient, predictable, and safe for patients. Ideal ortho-dontic movement occurs when a 'light and continuous' force is applied to the teeth, and ideally, any plastics used for CAT would deliver a constant therapeutic force over a relatively broad deflection range [22].

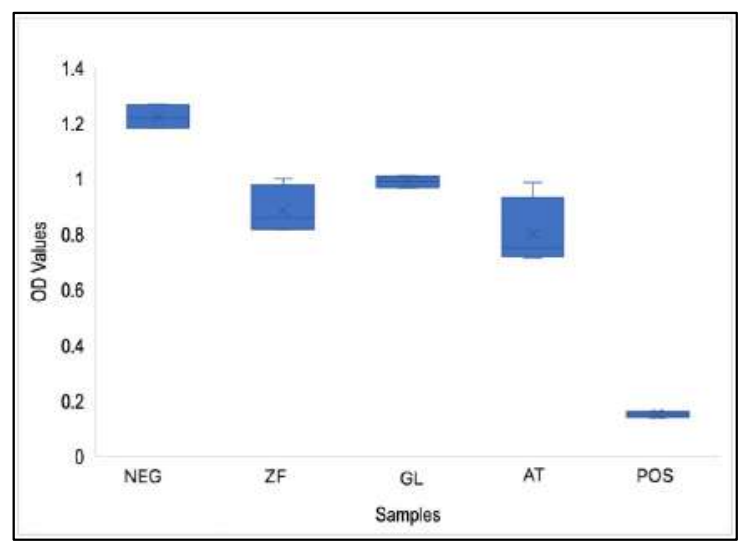

Figure 1. Optical Density of various materials used in the study

A study by Lombardo et al. in 2017 demonstrated that multilayer plastics initially display much less stiffness when compared to single-layered plastics, and their force decay showed far less deformation when prolonged stress was applied [23]. With such favourable force characteristics, there will certainly be more multilayered aligner materials available for retail in the future. Although several single-layer CAT materials have been shown to be safe in a clinical setting, there is little to no published evidence on the biocompatibility of these multilayered plastics at the time of the present study.

This study evaluated the in-vitro cytotoxicity of three different varieties of multilayered thermoplastic materials after 21 days of incubation in a simulated oral environment. Each sample extract was then exposed to a cell culture of HGFs, and the resultant cell viability was assessed. We chose gingival fibroblasts for our investigation because they are the most abundant cell type seen in the periodontal connective tissues, and they play a critical role in maintaining healthy gingival architecture [24]. They are commonly used in biocompatibility assessments of dental materials and are recommended for such assays by the International Standards Organization.

Mild levels of cytotoxicity were observed in all of the samples we tested, but none of them displayed levels that would contraindicate them for routine clinical use. This cytotoxic effect was similar to, and in some cases even lower, than that what has been observed in other orthodontic materials such as elastic ligatures, bonding materials, and molar bands [15,25,28]. Our findings align with the studies performed by Martina et al. (2019), who discovered that the thermoforming process may actually increase the cytotoxicity of each material when compared to their non-thermo-formed counterparts [18]. Conversely, other studies that have evaluated the biocompatibility of clear aligner materials found little to no negative effects in the past. An investigation of potential cytotoxicity and estrogenicity of Invisalign appliances by Eliades et al. (2009) revealed a complete absence of toxic effects, although it must be noted that this study was performed before the multilayered SmartTrack material was commercially launched [16,26]. Interestingly, the findings by Premaraj et al. (2014) showed similar cytotoxic results when samples were prepared in a saline solution, but cell viability significantly increased when exposed to a saliva-eluate; suggesting that saliva may offer protection against potentially harmful stimuli to the gingival tissues [17].

The cytotoxicity ranking system we used from Sjögren et al. [19] was further developed by Vande Vannet et al. in 2006 [20] by expounding the data from monolayer cell cultures and applying it to a three-dimensional epithelial model. They translated the [(1) noncytotoxic, (2) mildly cytotoxic, (3) moderately cytotoxic, and (4) markedly cytotoxic levels into a clinically applicable histological model. Their observations from the corresponding levels are as follows: (1) The epithelial tissues have a constant thickness, devoid of terminally differentiated cells (2) Minimal changes occur with slight edema. (3) The beginning of spongious tissue development in the upper layers with architectural atrophy and cellular irregularity. (4) Most of the upper cell layers of the epithelial tissues have disintegrated. There is cellular necrosis and loss of cellular junctions in the basal layer [20]. Using the data from Vande Vannet et al. (2006) [20], it is fair to extrapolate the present findings to determine that these materials are safe for clinical use. It must be acknowledged that our experimental conditions were not able to completely replicate the dynamic conditions encountered in the oral environment and therefore may be considered a limitation in the study. Variables in diet, hygiene, and salivary flow/composition can influence the chemical balance within the oral cavity. Mechanical forces from insertion, removal and non-nutritive mastication can potentially alter the physical properties of clear aligner materials in vivo [23]. Comparable in vitro experiments under similar conditions can offer viability to our study design and our current results, but further in vivo studies that take into consideration changes of $\mathrm{pH}$ and mechanical stresses of the oral cavity may improve experimental findings.

\section{Conclusion}

- In our experiment, all tested clear aligner materials showed mild levels of cytotoxicity.

- There were significant differences in cytotoxicity levels between the control and test samples, but not between the test samples themselves.

- Since the materials only showed mild levels of cytotoxicity, they can be determined as safe for clinical use. 
Conflicts of interest: Authors declared no conflicts of interest.

\section{Financial support: None}

\section{References}

1. Alansari RA, Faydhi DA, Ashour BS, Alsaggaf DH, Shuman MT, Ghoneim SH, Linjawi AI, Marghalani HY, Dause RR, Adult perceptions of different orthodontic appliances. Patient Preference and Adherence 2019:13 2119-2128. https://doi.org/10.2147/PPA.S234449

2. Rosvall MS, Fields HW, Ziuchkovski JP, Rosenstiel SF, Johnston WM, Attractiveness, acceptability, and value of orthodontic appliances. Am J Orthod Dentofacial Orthop 2009;135:276.e1-276e12. https://doi.org/10.1016/j.ajodo.2008.07.011

3. Ziuchkovski JP, Fields HW, Johnston WM, Lindsey DT. Assessment of perceived orthodontic appliance attractiveness. Am J Orthod Dentofacial Orthop. 2008;133:S68-78.

https://doi.org/10.1016/j.ajodo.2006.07.025

4. Walton DK, Fields HW, Johnston WM, Rosenstiel SF, Firestone AR, Christensen JC. Orthodontic appliance preferences of children and adolescents. Am J Orthod Dentofacial Orthop. 2010;138:698.e1-698.e12. https://doi.org/10.1016/j.ajodo.2010.06.012

5. Buschang PH, Shaw SG, Ross M, Crosby D, Campbell PM. Comparative time efficiency of aligner therapy and conventional edgewise braces. Angle Orthod. 2014;84:391-396. https://doi.org/10.2319/062113-466

6. Cardoso PC, Espinosa DG, Mecenas P, Flores-Mir C, Normando D, Pain level between clear aligners and fixed appliances: a systematic review. Prog Orthod. 2020;21:3. https://doi.org/10.1186/s40510-019-0303-z

7. Bräscher AK, Zuran D, Feldmanmn Jr. RE, Benrath J, Patient survery on Invisalign treatment comparing the SmartTrack material to the previously used aligner material. J Orofac Orthop. 2016;77:432-438. https://doi.org/10.1007/s00056-016-0051-3

8. Wheeler T, Patel N, McGorray, Effect of aligner material on orthodontic tooth movement. Journal of Aligner Orthodontics 2017;1:21-27

9. Giancotti A, Greco M, Gianluca M Extraction treatment using Invisalign technique. Prog Orthod. 2006;7;32-43

10. Garnett BS, Mahood K, Nguyen M, Al-khateeb A, Liu S, Boyd R, Oh H, Cephalometric comparisons of adult anterior open bite treatment using clear aligners and fixed appliances. Angle Orthod. 2019;89:3-9.

https://doi.org/10.2319/010418-4.1

11. Bucci R, Rongo R, Levatè, Michelotti A, Barone $S$, Razionale AV, D'Antò, Thickness of orthodontic clear aligners after thermoforming and after 10 days of intraoral exposure: a prospective clinical study. Prog Orthod. 2019;20:36. https://doi.org/10.1186/s40510019-0289-6

12. Align Technologies Inc. (2019, January 29) Align technology announces record 2018 financial results [Press release]. Retrieved from http://investor.aligntech.com/news-releases/newsrelease-details/align-technology-announces-record2018-financial-results

13. Buljan ZI, Ribaric SP, Abram M, Ivankovic A, Spalk S, In vitro oxidative stress induced by conventional and selfligating brackets. Angle Orthod. 2012;82:340-345. https://doi.org/10.2319/061811-395.1

14. Rongo R, Valletta R, Bucci R, Rivieccio V, Galeotti A, Michelotti A, D'antò V, In vitro biocompatibility of nickeltitanium esthetic orthodontic archwires. Angle Orthod. 2016;86:789-795. https://doi.org/10.2319/100415-663.1

15. Ahrari F, Afshari JT, Poosti M, Brook A, Cytotoxcity of orthodontic bonding adhesives resins on human oral fibroblasts. Eur J Orthod. 2010;32:688-692. https://doi.org/10.1093/ejo/cjq019

16. Eliades T, Pratsinis H, Athanasiou AE, Eliades G, Kletsas D, Cytotoxicity and estrogenicity of Invisalign appliances. Am J Orthod Dentofacial Orthop 2009;136:100-103.

https://doi.org/10.1016/j.ajodo.2009.03.006

17. Premaraj T, Simet S, Beatty M, Premaraj S, Oral epithelial cell reaction after exposure to Invisalign plastic material. Am J Orthod Dentofacial Orthop. 2014;145:64-71. https://doi.org/10.1016/i.ajodo.2013.09.011

18. Martina S, Rongo R, Bucci R, Razionale AV, Valletta R, D'Antò $\mathrm{V}$, In vitro cytotoxicity of different thermoplastic materials for clear aligners. Angle Orthod. 2019;89:942945. https://doi.org/10.2319/091718-674.1

19. Sjögren G, Sletten G, Dahl J, Cytotoxicity of dental allowys, metals, and ceramics assess by Millipore filter, agar overlay, and MTT tests. J Prosthet Dent. 2000;82:229-236. https://doi.org/10.1067/mpr.2000.107227

20. Vande Vannet B, Mohebbian N, Wehrbein H. Toxicity of used orthodontic archwires assessed by three-dimensional cell culture. Eur J Orthod. 2006;28:426-432.

https://doi.org/10.1093/ejo/cj1002

21. Sasaki K, Tanaka N, Watanaba M, Yamada M. Comparison of cytotoxic effects of chemicals in for different cell types. Toxic. In Vitro. 1991;5/6:403-406. https://doi.org/10.1016/0887-2333(91)90061-H

22. Proffit WR, Fields HW, Sarver DM. Contemporary orthodontics. 4th ed. St. Louis: Mosby; 2007.

23. Lombardo L, Martines E, Mazzanti V, Arreghini A, Mollica F, Siciliani G; Stress relaxation properties of four orthodontic aligner materials: a 24-hour in vitro study. Angle Orthod. 2017; 87:11-18. https://doi.org/10.2319/113015-813.1

24. Lee Ik, Lee $\mathrm{Mj}$, Jang Hs. The interrelationship between human gingival fibroblast differentiation and cultivating time. Tissue Engineering Regenerative Medicine. 2013;10: 60-64. https://doi.org/10.1007/s13770-013-0371-y

25. D’Antó V, Spagnuolo G, Polito I, Paduano S, Ambrosio L, Valletta R. In vitro cytotoxicity of orthodontic primers. Prog Orthod. 2009;10:4-11

26. Align Technologies. (2017, May 24) Align Technology Receives U.S. Patents for SmartTrack(R) Invisalign(R) Aligner Material [Press release]. https://investor.aligntech.com/static-files/2fded79c-c37e47e6-b7d3-25e43c96f744

27. Schuster S, Eliades G, Zinelis S, Eliades T, Bradley G. Structural conformation and leaching from in vitro aged and retrieved Invisalign appliances. Am J Orthod Dentofacial Orthop.2004;136:725-728. https://doi.org/10.1016/j.ajodo.2004.04.021

28. Goncalves TS, Menezes LM, Trindade C, Machado MS, Thomas P, Fenech M, Henriques J. Cytotoxicity and genotoxicity of orthodontic bands with or without silver soldered joints. Mutation Research/Genetic Toxicology and Environmental Mutagenesis. 2014;76:21-8. https://doi.org/10.1016/i.mrgentox.2014.01.011

29. Stockert J, Horobin R, Colombo L, Blazquez-Castro A. Tetrazolium salts and formazan products in Cell Biology: Viability, flouresence imaging, and labeling perspectives. Acta Histochemica. 2018;120:159-167. 
https://doi.org/10.1016/j.acthis.2018.02.005

30. Berridge $\mathrm{M}$, Herst $\mathrm{P}$, Tan A. Tetrazolium dyes as tools in cell biology: new insights into their cellular reduction. Biotechnology Annual Review. 2005;11:127-152.

https://doi.org/10.1016/S1387-2656(05)11004-7

How to cite this article: Marsh S, Anthony R, Barnett B, Shou Ch, Saunders K. Comparison between the in-vitro cytotoxicity of three different multilayer thermoplastic clear aligner materials. Int $\mathrm{J}$ Dent Mater. 2022; 4(1): 1-5.

DoI: http://dx.doi.org/10.37983/IJDM.2022.4101 